\title{
Microscale AMPAR Reorganization and Dynamics of the Postsynaptic Density
}

\author{
Sandra Jurado ${ }^{1}$ and Shira Knafo ${ }^{2}$ \\ ${ }^{1}$ Nancy Pritzker Laboratory, Department of Psychiatry and Behavioral Sciences, Stanford University School of Medicine, Palo Alto, California, 94304, and \\ ${ }^{2}$ Centro de Biología Molecular "Severo Ochoa," Consejo Superior de Investigaciones Científicas/Universidad Autónoma de Madrid, 28049 Madrid, Spain \\ Review of Kerr and Blanpied
}

AMPA-type receptors (AMPARs) are glutamate-gated channels whose postsynaptic activation convey the major depolarization in brain excitatory neurotransmission. Trafficking of these receptors to and from synapses is tightly regulated in neurons and underlies long-lasting forms of synaptic plasticity. For example, export of AMPARs from the endoplasmic reticulum to the Golgi (Vandenberghe and Bredt, 2004) is suggested to contribute to the expression of certain types of synaptic plasticity (Broutman and Baudry, 2001). In addition, endocytosis removes AMPARs from synapses during LTD (Beattie et al., 2000) and in response to other stimuli (Man et al., 2000). Internalized AMPARs can be degraded in lysosomes or recycled back to the surface membrane (Ehlers, 2000; Gruenberg, 2001). This AMPAR sorting is regulated by synaptic activity (Ehlers, 2000) and provides the local intracellular pool of AMPARs needed for LTP expression (Park et al., 2004). AMPARs also undergo consti-

\footnotetext{
Received March 2, 2012; revised April 4, 2012; accepted April 4, 2012

This work was supported by a grant from the Spanish Ministry of Science and Innovation (SAF2010-15676 to S.K.). S.K. is the recipient of a "Ramón y Cajal" contract from the Spanish Ministry of Science and Innovation. We thank Prof. Jose A. Esteban for commenting on the manuscript.

Correspondence should be addressed to either of the following: Dr. Sandra Jurado, Nancy Pritzker Laboratory, Department of Psychiatry and Behavioral Sciences, Stanford University School of Medicine, Palo Alto, CA 94304, E-mail: sjurado@stanford.edu; or Dr. Shira Knafo, Centro de Biología Molecular "Severo Ochoa," Consejo Superior de Investigaciones Científicas/Universidad Autónoma de Madrid, 28049 Madrid, Spain, E-mail: sknafo@cbm.uam.es.

DOI:10.1523/JNEUROSCI.1048-12.2012

Copyright $\odot 2012$ the authors $\quad 0270-6474 / 12 / 327103-03 \$ 15.00 / 0$
}

tutive trafficking that involves both exocytic delivery from intracellular compartments (Gerges et al., 2006) and fast exchange with surface extrasynaptic receptors through lateral diffusion (Tardin et al., 2003). Still, knowledge is lacking regarding the organization and regulation of AMPARs within the postsynaptic density (PSD) and the events triggering their repositioning.

AMPAR trafficking can be evaluated by biochemical, electrophysiological, and imaging approaches. Synaptic delivery of endogenous AMPARs can be monitored by measuring levels of specific AMPAR subunits in synaptoneurosomes (a fraction of brain extracts enriched in synaptic elements) (Heynen et al., 2000). To track specifically the movement of endogenous AMPARs on the cell surface, rapid timelapse imaging of individual semiconductor quantum dots coupled to AMPAR antibodies are performed (Dahan et al., 2003). Overexpression of GluAl-GFP or GluA2 (R586Q)-GFP AMPAR subunits allows visualization of recombinant AMPARs to detect general distribution and movement. This overexpression results in formation primarily of homomeric AMPARs that have different conductance properties than endogenous AMPARs (Shi et al., 2001). This "electrophysiological tagging" is a powerful tool to detect trafficking of AMPARs into synapses, yet it does not identify movements of AMPARs within synapses.

The past decades have witnessed a remarkable increase in the application of fluorescence recovery after photobleach- ing (FRAP) (Jacobson et al., 1976) to quantify the dynamics of proteins and lipids within a defined subcellular compartment. In this assay, fluorescent molecules are irreversibly photobleached in a small area of the cell by a focused laser beam. Subsequent diffusion of surrounding nonbleached molecules into the bleached area leads to a total or partial recovery of fluorescence that is proportional to the mobility of a given molecule under different experimental conditions (Fig. 1). However, with regular FRAP, it is technically challenging to pinpoint subcellular AMPAR movement within living synapses with sufficient temporal and spatial resolution.

The study by Kerr and Blanpied (2012) overcame these technical limitations using high-resolution photobleaching of recombinant fluorescent receptors on the surface of single spines. To view exclusively surface AMPARs (but not the receptors in intracellular compartments) the authors used primary neurons expressing GluA1 and GluA2 AMPAR subunits fused to the $\mathrm{pH}$-sensitive GFP [Super Ecliptic pHluorin (SEP)], which does not fluoresce in the acid environment of intracellular compartments. In addition, Kerr and Blanpied (2012) took advantage of the fact that in primary hippocampal neurons, GluA1-GFP subunits spontaneously concentrate at synapses. This is in contrast to neurons in organotypic slice cultures in which GluA1-GFP subunits distribute diffusely throughout 


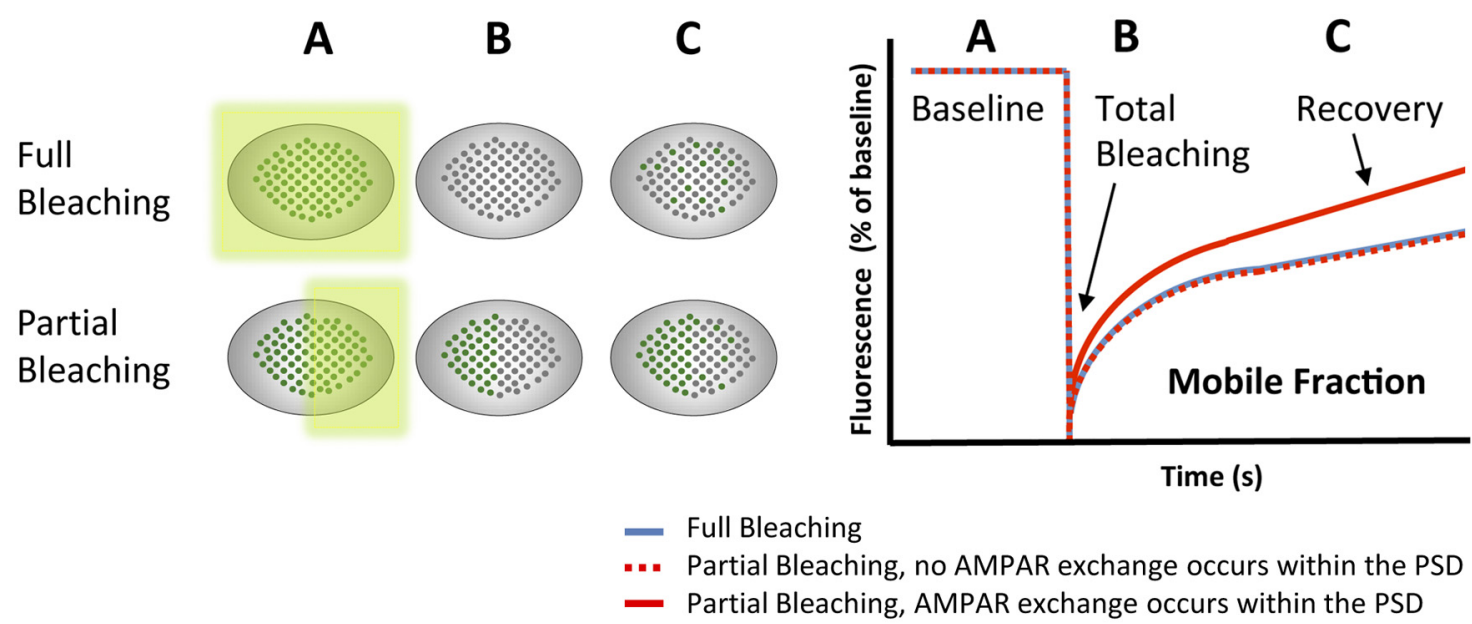

Figure 1. Principles of FRAP experiment with AMPARs. Left, Scheme illustrating photobleaching and recovery of a whole synapse (top, Full Bleaching) versus half of a synapse (bottom, Partial Bleaching). Before the bleach event, fluorescent AMPARs can be viewed on the synaptic surface ( $A$, green dots, baseline). Immediately after photobleaching, AMPARs are no longer fluorescent (B, gray dots, total bleaching) and then fluorescence gradually recovers ( $C$, green and gray dots, recovery) as unbleached AMPARs move into the bleached area. Note that, under basal conditions, full bleaching and partial bleaching result with the same recovery graph (right, blue line and dashed red line, respectively). When intrasynaptic mobility of AMPARs is increased (e.g., after glutamate application), there is a stronger increase in recovery following partial bleaching (right, solid red line).

the dendritic tree and require LTP-like events to efficiently enter into dendritic spines (Shi et al., 1999). Therefore, in primary neurons, recombinant AMPARs at synapses can be viewed without preceding manipulations. With these methods, the authors demonstrated the use of FRAP as a practical and reproducible method to study AMPARs repositioning within the PSD.

Kerr and Blanpied (2012) first aimed to elucidate whether, under basal conditions, AMPARs diffuse laterally within the PSD of single spines. They found that the fluorescence recovery curve in synapses that were entirely photobleached (Fig. 1, Full Bleaching) was similar to the curve of synapses in which only a subdomain was bleached (Fig. 1, Partial Bleaching), implying that no AMPAR exchange occurred within the PSD. This is in agreement with previous studies demonstrating restricted diffusion of AMPARs within individual synapses (Tardin et al., 2003; Makino and Malinow, 2009). Thus, the postsynaptic scaffolding matrix significantly restricts the redistribution of AMPARs within the synapse. It is, however, possible that the overexpression of AMPAR subunits (also leading to formation of homomeric receptors in nonphysiological levels, instead of the natural heteromeric receptors) physically restricts their own mobility.

Kerr and Blanpied (2012) hypothesized that AMPAR distribution within the PSD depends on their association with specific postsynaptic scaffold proteins. They determined the degree of this association by calculating the pixel-wise fluorescence correlation coefficient $\left(R_{\mathrm{F}}\right)$ for
PSD-95, GKAP, Shank, and Homer, all of which are postsynaptic scaffolding proteins. A high $R_{\mathrm{F}}$ between a scaffold protein and AMPARs at individual spines indicated they had similar subsynaptic distributions. The highest $R_{\mathrm{F}}$ was found between AMPARs and PSD-95, although the $\mathrm{C}$ termini of AMPA receptor subunits do not directly bind to this scaffolding protein. Nevertheless, this tight colocalization may account for the crucial role PSD-95 has in controlling the number of synaptic AMPARs (Schnell et al., 2002).

The immobility of receptors within the PSD led Kerr and Blanpied (2012) to examine whether the overall structure of individual AMPAR clusters is rigid over time. To this end, the authors performed extended $(1 \mathrm{~h})$ time-lapse imaging of synaptic clusters composed of surface AMPARs. As expected from previous studies showing a substantial PSD flexibility (Blanpied et al., 2008), they observed that individual AMPAR clusters exhibit substantial and continuous changes in their morphology. In contrast to the continuously dynamic structure of AMPAR clusters, SEP fluorescence intensity was extremely stable over time. These results imply that the structural flexibility of AMPAR clusters is not accompanied by significant changes in the number of surface receptors.

Actin, a cytoskeletal protein highly enriched in dendritic spine heads, where it is thought to anchor AMPARs, was an obvious candidate for the control of the observed reshaping of AMPAR clusters and perhaps for AMPAR retention within the PSD. Remarkably, both preventing actin monomer assembly into filaments (with latrunculin) and stabilizing actin polymerization (with jasplakinolide) transformed the AMPAR clusters into absolutely rigid structures. This finding suggests that constitutive reshaping of the synaptic AMPAR clusters requires ongoing actin turnover. Contrary to some predictions, acute application of latrunculin did not increase AMPAR loss from the synapse nor did it affect intrasynaptic receptor mobility, as discovered by subdomain FRAP. These are important findings, because they suggest that actin treadmilling is not acutely necessary for AMPAR synaptic retention or mobility, challenging the notion that actin anchors AMPARs at synapses.

To test whether AMPAR activation promotes internal AMPAR repositioning, Kerr and Blanpied (2012) applied glutamate to cultured neurons. This manipulation induced a significant increase in the intrasynaptic mobility of AMPARs that became evident when only a subdomain of the spine was photobleached (Fig. 1). This suggests that activated synapses increase their exchange rate of receptors among different subdomains. These results are consistent with the notion that the PSD acts as a network that regulates subsynaptic receptor distribution so receptors can respond with high efficacy to glutamate release (Elias and Nicoll, 2007). Does intrasynaptic receptor mobility increase during LTP as well? A hint for this question can be found in a recent study (Makino and Malinow, 2009) using similar approaches (i.e., expression of fluorescent receptors in organotypic hippocampal slices combined with FRAP and glutamate un- 
caging). Makino and Malinow (2009) suggested that the mobility of SEPGluA1 subunits is significantly decreased after LTP induction to preserve the recently requited receptors and maintain synaptic potentiation.

In summary, the study of Kerr and Blanpied (2012) represents an important advance in the study of the microscale organization and dynamics of the postsynaptic membrane. Using FRAP on subdomains of spines in dissociated neurons, they determined that AMPARs are relatively immobile within the PSD while displaying an overall motion as clusters in a matrix that constantly reshapes in an actin-dependent manner. Their conclusions challenge previous models for AMPA receptor positioning and anchoring at the postsynaptic density, and offer critical insight into the inner organization of living synapses. Undoubtedly, the final picture of AMPAR trafficking will require the combination of complementary imaging techniques. In the next years, FRAP will probably be combined with the use of photo-switchable fluorescent proteins (fluorescent proteins that change their excitation and emission spectra when exposed to specific light) to explore receptor mobility, and with high spatial and temporal resolution imaging, such as Photoactivated Localization Microscopy and stochastic optical reconstruction microscopy.

\section{References}

Beattie EC, Carroll RC, Yu X, Morishita W, Yasuda H, von Zastrow M, Malenka RC (2000) Regulation of AMPA receptor endocytosis by a signaling mechanism shared with LTD. Nat Neurosci 3:1291-1300.

Blanpied TA, Kerr JM, Ehlers MD (2008) Structural plasticity with preserved topology in the postsynaptic protein network. Proc Natl Acad Sci U S A 105:12587-12592.

Broutman G, Baudry M (2001) Involvement of the secretory pathway for AMPA receptors in NMDA-induced potentiation in hippocampus. J Neurosci 21:27-34.

Dahan M, Lévi S, Luccardini C, Rostaing P, Riveau B, Triller A (2003) Diffusion dynamics of glycine receptors revealed by singlequantum dot tracking. Science 302:442-445.

Ehlers MD (2000) Reinsertion or degradation of AMPA receptors determined by activitydependent endocytic sorting. Neuron 28: 511-525.

Elias GM, Nicoll RA (2007) Synaptic trafficking of glutamate receptors by MAGUK scaffolding proteins. Trends Cell Biol 17:343-352.

Gerges NZ, Backos DS, Rupasinghe CN, Spaller MR, Esteban JA (2006) Dual role of the exocyst in AMPA receptor targeting and insertion into the postsynaptic membrane. EMBO J 25:1623-1634.

Gruenberg J (2001) The endocytic pathway: a mosaic of domains. Nat Rev Mol Cell Biol 2:721-730.

Heynen AJ, Quinlan EM, Bae DC, Bear MF (2000) Bidirectional, activity-dependent regulation of glutamate receptors in the adult hippocampus in vivo. Neuron 28:527-536.

Jacobson K, Derzko Z, Wu ES, Hou Y, Poste G (1976) Measurement of the lateral mobility of cell surface components in single, living cells by fluorescence recovery after photobleaching. J Supramol Struct 5:565(417)-576(428).

Kerr JM, Blanpied TA (2012) Subsynaptic AMPA receptor distribution is acutely regulated by actin-driven reorganization of the postsynaptic density. J Neurosci 32:658-673.

Makino H, Malinow R (2009) AMPA receptor incorporation into synapses during LTP: the role of lateral movement and exocytosis. Neuron 64:381-390.

Man HY, Ju W, Ahmadian G, Wang YT (2000) Intracellular trafficking of AMPA receptors in synaptic plasticity. Cell Mol Life Sci 57:1526-1534.

Park M, Penick EC, Edwards JG, Kauer JA, Ehlers MD (2004) Recycling endosomes supply AMPA receptors for LTP. Science 305:1972-1975.

Schnell E, Sizemore M, Karimzadegan S, Chen L, Bredt DS, Nicoll RA (2002) Direct interactions between PSD-95 and stargazin control synaptic AMPA receptor number. Proc Natl Acad Sci U S A 99:13902-13907.

Shi SH, Hayashi Y, Petralia RS, Zaman SH, Wenthold RJ, Svoboda K, Malinow R (1999) Rapid spine delivery and redistribution of AMPA receptors after synaptic NMDA receptor activation. Science 284:1811-1816.

Shi S, Hayashi Y, Esteban JA, Malinow R (2001) Subunit-specific rules governing AMPA receptor trafficking to synapses in hippocampal pyramidal neurons. Cell 105:331-343.

Tardin C, Cognet L, Bats C, Lounis B, Choquet D (2003) Direct imaging of lateral movements of AMPA receptors inside synapses. EMBO J 22:4656-4665.

Vandenberghe W, Bredt DS (2004) Early events in glutamate receptor trafficking. Curr Opin Cell Biol 16:134-139. 